Meta

Journal des tradlucteurs

Translators' Journal

\title{
La traduction de l'aspect verbal français en igbo
}

\section{Benedict O. Okiwelu}

Volume 36, numéro 2-3, juin 1991

URI : https://id.erudit.org/iderudit/003760ar

DOI : https://doi.org/10.7202/003760ar

Aller au sommaire du numéro

Éditeur(s)

Les Presses de l'Université de Montréal

ISSN

0026-0452 (imprimé)

1492-1421 (numérique)

Découvrir la revue

Citer cet article

Okiwelu, B. O. (1991). La traduction de l'aspect verbal français en igbo. Meta, 36(2-3), 471-483. https://doi.org/10.7202/003760ar d'utilisation que vous pouvez consulter en ligne.

https://apropos.erudit.org/fr/usagers/politique-dutilisation/ 


\section{LA TRADUCTION DE L'ASPECT VERBAL FRANÇAIS EN IGBO}

BENEDICT O. OKIWELU

University of Calabar, Calabar, Nigeria

Au Nigeria, à cause de notre expérience coloniale, nous sommes habitués quotidiennement à traduire de l'anglais en langue maternelle et inversement. Même au niveau universitaire où les cours de traduction occupent une place importante dans les programmes d'étude, l'axe traditionnel est anglais-français-anglais. Il s'avérerait pour ainsi dire bizarre, voire insolite, à première vue, que d'assister à la traduction du français en igbo, langue presque entièrement inconnue à l'échelle internationale. Pourtant, l'intérêt croissant que suscite actuellement l'étude de la langue igbo chez des linguistes autochtones et étrangers est à noter. Outre les ouvrages de grammaire et de littérature igbo qui sont en train de paraître, il y a, à l'heure actuelle, des spécialistes français qui travaillent sur la langue igbo aussi bien personnellement qu'à titre officiel dans des universités nigériannes et même à Paris. La langue igbo elle-même est parlée par environ 14 millions d'habitants de l'ethnie igbo dont la population est concentrée essentiellement dans la région est du Nigeria et partiellement sur le côté ouest du même fleuve Niger qui traverse le pays. Parmi les quelque 395 parlers locaux nigérians, l'igbo a été constitutionnellement désignée l'une des trois langues nationales (le hausa et le yoruba étant les deux autres) qui serviront côte à côte avec l'anglais, espérons-le, de véhicule de délibération à l'assemblée nationale dès le retour au gouvernement civil. La traduction de l'aspect verbal français en igbo contribuera à la découverte de même qu'à la mondialisation de cette langue qui est toujours en voie de développement. Ce travail nous permettra aussi d'apprécier le système aspectuel des deux langues dont le contact est insuffisamment connu jusqu'ici. Sans doute, deux langues humaines si éloignées soient-elles structuralement et génétiquement l'une de l'autre auront quand même des points de rencontre au niveau de la communication. Ce truisme est confirmé par Georges Mounin (1963:42) lorsqu'il dit:

Toutes les langues devaient communiquer les unes avec les autres parce qu'elles parlaient, toutes et toujours, du même univers de la même expérience humaine, analysé selon des catégories de la connaissance idéologique pour tous les hommes.

Pour bien vérifier cette hypothèse, notre première tâche, celle d'investigation, consistera à voir si cette catégorie grammaticale existe en igbo et à quel point. Deuxièmement, nous verrons s'il y a des obstacles linguistiques qui gênent le passage de l'aspect verbal depuis la langue source jusqu'à la langue cible, et finalement, nous trouverons un mécanisme susceptible d'enlever les obstacles.

\section{L'ASPECT VERBAL FRANÇAIS EST-IL TRADUISIBLE EN IGBO?}

En d'autres termes, existe-t-il l'expression aspectuelle en igbo ? Pour répondre à cette question, il sera pertinent de voir d'abord comment l'aspect verbal français s'exprime.

La Grammaire Larousse (1964: 328) répartit l'aspect verbal français en deux grandes catégories formelles, d'une part, en formes grammaticales et, d'autre part, en formes périphrastiques. Les formes grammaticales sont réparties à leur tour en trois souscatégories: forme simple, qui se construit avec un seul élément verbal et qui exprime 
l'aspect inaccompli, et forme composée, se traduisant par deux éléments verbaux (auxiliaires avoir ou être + participe passé) et qui exprime l'aspect accompli, forme surcomposée (double auxiliaire $+p$. passé) qui traduit l'aspect doublement accompli. Les formes périphrastiques (semi-auxiliaire + infinitif ou $p$. présent) expriment les autres moments du déroulement de l'action. L'un des défauts de ce classement, pourtant intéressant, est que les autres moments du déroulement sont limités à quatre phases seulement au lieu de cinq. De même qu'elle passe sous silence les formes affixales et adverbiales de l'aspect. Tout en suivant ce classement, nous allons l'élargir en ajoutant ces formes qui y manquent. Il reste maintenant à formuler des phrases type et à les traduire pour voir si elles ont des équivalents en igbo. Pour délimiter notre champ d'opération et donc réaliser notre objectif, il ne sera pas tenu compte d'aspect issu de la voix passive. En igbo, tous les tons: /(ton haut), \(ton bas), - (ton haut abaissé), seront notés, sauf ce premier. La variante utilisée ici est l'igbo d'Onicha (orthographe coloniale, Onitsha) puisque son orthographe est plus stable que celle de l'igbo central qui est toujours en voie de standardisation.

1.1 L'aspect simple (aspect inaccompli)

1.1.1 Pierre lit un livre.

1.1.2 Le fusil éclate.

1.1.3 Pierre lisait un livre.

Pità nà àgu akwrikwo.

Egbè àgbaa.

Pità nàbù àgu akwukwọ.

1.2 Forme composée (aspect accompli)

1.2.1 Pierre a lu un livre.

1.2.2 Marie est allée au marché.

Pità àgugo akwukwo. Marià èjego afia.

1.3 Forme surcomposée (aspect doublement accompli)

1.3.1 Quand Pierre a eu lu un livre, il est allé au marché. Ogè Pità gùsili akwukwo, o jèli afia.

1.4 Formes périphrastiques

Ce sont les cinq phases suivantes:

1.4.1 Phase $\mathrm{A}$, indiquant le déroulement prochain du procès : Pierre va lire un livre.

Pità gàfu àgu akwukwọ.

Pierre est sur le point de lire un livre

1.4.2 Phase $\mathrm{B}$, indiquant le début du procès: Pierre commence à lire un livre.

Pierre se met à lire un livre.

1.4.3 Phase C, indiquant l'action en cours:

Pierre est en train de lire un livre.

Le petit chat va grelottant.

Pità gàfu àgu akwikiwo.

1.4.4 Phase $\mathrm{D}$, indiquant la fin du procès :

Pierre finit de lire un livre.

Pità àguba akwukwọ.

Pità àguba akwuikwọ.

Pità nà agu aswrkwo.

Nwà̀bàrà nà àma jîjijì.

1.4.5 Phase $\mathrm{E}$, indiquant le moment qui succède à la fin du procès:

Pierre vient de lire un livre.

Pità gùfuu akwukwọ.

\subsection{La forme affixale}

Elle est marquée soit par le préfixe, ex. re- (aspect itératif):

1.5.1 Pierre relit un livre.

Pità nà àguzi akwukwo.

- soit par l'infixe, ex. -ll- (aspect fréquentatif):

1.5.2 Les vautours tiraillent la charogne.

Ùdènè nà àdocha ozu anù. 


\subsection{Forme de marque adverbiale}

Le marqueur peut être un adverbe, ex. toujours (progressif renforcé):

1.6.1 Pierre lit toujours.

Pità nà àgulili akwukwọ.

ou bien une locution adverbiale, ex. plusieurs fois (multiplicatif) :
1.6.2 Pierre lit plusieurs fois.
Pità nà àgukata $a k w u k w o ̣$.

À partir des exemples et traduction ci-dessus, il est clair que nous avons pu exprimer en igbo les idées véhiculées en français, c'est-à-dire l'inaccompli et les accomplis aussi bien que d'autres types du déroulement. Cette possibilité vient du fait qu'il existe également l'expression aspectuelle en igbo. Elle est due aussi à quelque parallélisme structural entre les deux langues. Linguistiquement, les deux langues expriment l'aspect au moyen du verbe. Elles se servent également de phrases qui suivent l'ordre sujetverbe-complément. Troisièmement, les deux langues font la distinction formelle entre les différents types d'aspect. En dépit de ces similitudes, il existe une grande divergence structurale entre les deux. En fait, la traduction des phrases-sources n'a été possible que grâce à la compétence linguistique du traducteur, à son bilinguisme, plutôt qu'au parallélisme indiqué plus haut. Or, la répartition de l'aspect selon le critère formel et à laquelle on vient d'assister ne regarde que la langue-source. Cette méthode ne permet pas de voir ce qui se passe structuralement dans la langue-cible ni d'y passer clairement le message de la langue-source. Même à l'intérieur du camp français la méthode est encore défectueuse. Elle établit une ligne de démarcation entre les formes dites grammaticales et les formes périphrastiques sans chercher à trouver un point de rencontre quel qu'il soit entre les deux groupes. Pourtant, il est évident que les formes exprimant les autres moments de l'aspect peuvent quelquefois être grammaticalisées pour donner en quelque sorte un accompli subjectif. Ces deux facteurs, divergence structurale et méthode de classement, rendent inévitables les obstacles linguistiques au niveau de la traduction. Nous allons les exposer.

\section{LES OBSTACLES LINGUISTIQUES}

La nature des obstacles linguistiques réside dans l'impossibilité d'une traduction mot à mot, car l'expression de l'aspect français n'opère pas le même système lexicogrammatical que celui de l'igbo même au niveau de la même idée. Les obstacles sont de sept types. Ils seront énumérés au fur et à mesure.

\subsection{L'obstacle morphologique}

Le système morphologique d'aspect français ne saurait être transféré directement à celui de l'igbo, les deux étant différents. Pour l'aspect inaccompli, le français utilise un seul élément verbal tandis que l'igbo utilise soit un élément verbal soit deux, selon le sens $\mathrm{du}$ verbe (voir respectivement 1.1.2 et 1.1.1). Inversement, le français utilise deux éléments verbaux (aux. + p. passé) pour traduire l'aspect accompli (cf. 1.2.1 et 1.2.2) et trois éléments verbaux (aux. + aux. + p.p.) pour l'aspect doublement accompli (1.3.1); mais l'igbo se sert d'un seul élément verbal pour traduire chacune des deux parcelles aspectuelles. De plus, la détermination de l'aspect inaccompli ou de l'aspect accompli en français est foncièrement grammaticale. Les morphèmes marqueurs d'aspect sont à caractère désinentiel. Ainsi, le verbe simple aussi bien que l'auxiliaire varient en nombre et en personne selon le sujet, de même que le participe passé fléchit en nombre et genre quand il est auxilié par être comme dans 1.2.2 et même par avoir avec un complément d'objet artéposé. Les verbes igbo n'ont pas de variantes désinentielles, ni de genre. Les nombre et personne du verbe sont marqués par le pronom sujet. Pour exprimer l'aspect en igbo, les verbes se servent de morphèmes suffixaux à ton haut. Seul l'auxiliaire est à ton bas. Cela revient à dire que les morphèmes suffixaux qui sont à ton bas sont d'ordre temporel 
(vide 1.1.3 et 1.3.1). Même lorsque les deux langues usent de la même forme morphologique, la convergence n'est qu'apparente. Ainsi, la marque aspectuelle par la forme suffixale (1.5) est réalisée en français au niveau d'infixe et de préfixe mais suffixale en igbo. Ces différences morphologiques rendent impossibles la traduction littérale.

\subsection{L'obstacle morpho-sémantique}

Le système morphologique de l'aspect français est sémantiquement complexe. L'idéal serait qu'à chaque signifiant aspectuel corresponde un seul signifié. Malheureusement, ce n'est pas toujours le cas. Nous venons de signaler que l'aspect simple en français est traduit en igbo par deux formes différentes: un élément verbal dans un cas (àgbaa 1.1.2) et deux éléments dans un autre ( $n a ̀$ àgu 1.1.1). Il en est ainsi puisqu'il existe un obstacle morpho-sémantique dans la forme aspectuelle française bien avant la traduction: le français se sert d'une seule forme verbale pour exprimer deux valeurs aspectuelles différentes, comme le montre encore l'exemple suivant:

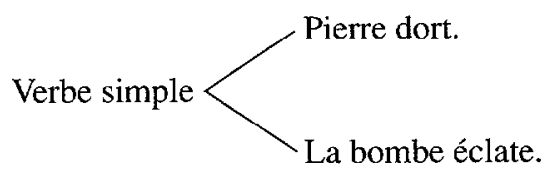

(Aspect progressif)

(Aspect momentané)

qui se traduit par

$$
\begin{aligned}
& \text { Verbe composé - Pità nà àlaru ulă. (Aspect progressif) } \\
& \text { Verbe simple - Bombu àgbaa. (Aspect momentané) }
\end{aligned}
$$

étant entendu que «Pierre dort» est l'équivalent sémantique de «Pierre est en train de dormir» et que «Pierre est en train de dormir» et que «La bombe est en train d'éclater» sont grammaticalement inacceptables. De même que dans «Pierre relit un livre» (cf. 1.5.1), le verbe renferme deux valeurs aspectuelles, itératives, marquées par le morphème re-, et progressives, marquées implicitement par le lexème verbal. Il en va de même pour tirailler dans l'exemple 1.5.2 qui est à la fois fréquentatif et progressif pour la même raison. Cette ambiguiité n'existe pas en igbo qui marque ici les deux distinctions formelle et sémantique. C'est qu'en igbo l'expression aspectuelle dépend ici du sémantisme du lexème verbal tandis qu'en français la valeur d'inaccompli est subordonnée à la forme verbale. Un problème analogue persiste au niveau des formes composée et surcomposée. Pour la composée, un seul bloc morphologique (aux. + p.p.) renvoie à deux valeurs sémantiques différentes: aspect et temps. Ainsi, les phrases 1.2.1 et 1.2.2 que nous reprenons ici peuvent chacune se traduire en igbo de deux manières différentes:

Pità àgubo akurukwo.

Pierre a lu un livre.

Pità gùlù akưkwo.

Marià èjego afia.

Marie est allée au marché.

Marià jèli afía.
(Aspect accompli)

(Temps parfait)

(Aspect accompli)

(Temps parfait)

à moins qu'il y ait dans l'entourage verbal un modificateur temporel pour préciser que la phrase à traduire insiste sur le temps et non sur l'aspect. Quant à l'exemple 1.3.1, le même problème se pose. La forme morphologique surcomposée exprime tour à tour l'aspect accompli et le passé antérieur. Seules la conjonction «quand» et l'opposition de 
«a eu lu» et «est allé» permettent de savoir que l'action de la première combinatoire est antérieure à celle de la deuxième et qu'elle met, par conséquent, l'accent sur le temps. Toutes ces ambiguités montrent qu'il est difficile de traduire directement en igbo les éléments morphologiques français.

\subsection{L'obstacle lexical}

La traduction des autres moments du déroulement de l'action française n'aboutit pas forcément à la même quantité lexicale en igbo. Bien que le français exprime ce genre d'aspect au moyen de la forme périphrastique qui mène au moins à deux verbes différents, l'igbo le traduit par le système de suffixation, ce qui donne un seul verbe:
2.3.1 Il commence à lire.
12
O guba.

ou à deux éléments verbaux :

2.3.2 Il va commencer à finir de lire. $\begin{array}{llll}1 & 2 & 3 & 4\end{array}$<smiles>[14CH2][14CH2][14CH2][14C](=O)I</smiles>

Le traducteur non igbophone risque de commettre l'erreur de suivre la structure lexico-analytique française de la phrase 2.3 .2 en la traduisant mot à mot pour obtenir bon gré mal gré la même quantité lexicale en igbo telle que :

o gàfu èbido ikwusi idesini,

une traduction qui, tout en n'étant pas incorrecte, est pourtant grammaticalement inférieure à notre équivalent synthétique. L'erreur dans laquelle est susceptible de glisser le traducteur non igbophone est celle de traduction peu idiomatique. Par ailleurs, la traduction lexicale de l'aspect français peut ne pas se limiter au verbe; elle peut se réaliser des fois par verbe + adverbe (cf. 1.6). L'erreur de traduction consisterait à chercher coûte que coûte les adverbes en igbo qui sont presque inexistants d'ailleurs.

\subsection{L'obstacle lexico-sémantique}

Le système lexical de l'aspect français peut provoquer une ambiguïté d'interprétation et rendre ainsi difficile la traduction. Dans l'exemple ci-après, le bloc semi-auxiliaire + inf. aboutit à trois rendements différents en igbo.

Pità gà àfu nnà ya.

Pierre va voir son père.

Pità gà èje ifü nnà ya.
(Futur proche)

Pità gàfu àfu nnà ya. (Aspect imminent) (Mouvement)

Ces interprétations différentes en igbo sont dues à la complexisation sémantique de l'auxiliaire aller qui renvoie à trois valeurs sémantiques différentes, lesquelles peuvent être également celles :

i) de temps:

Pierre verra son père tout à l'heure;

ii) d'aspect: Pierre est sur le point de voir son père;

iii) de mouvement: Pierre se dirige vers son père pour le voir.

Le traducteur n'a qu'à choisir entre les trois possibilités; mais son choix peut ne pas être le bon. Inversement, il arrive qu'un élément lexical igbo prête à l'ambiguïté d'interprétation. C'est le cas de l'auxiliaire nà qui exprime à la fois l'aspect progressif et l'aspect habituel: 
Pierre est en train d'écrire. (progression)

$$
+
$$

Pierre a l'habitude d'écrire.

$$
=
$$

Pità nà ède akwukwo. (progression + habitude)

Pierre va à l'école tous les jours. Pità nà èje akwukwo ubosi nine.

$$
\text { (habitude) (progression }+ \text { habitude) }
$$

L'erreur de traduction consisterait à rechercher à tort et à travers un auxiliaire habituel qui serait différent de celui du progressif.

\subsection{L'obstacle morpho-syntactique}

La combinatoire des éléments morphologiques d'aspect ne suit pas la même régularité dans les deux langues. Par exemple, pour exprimer l'aspect accompli, la présence des morphèmes aspectuels du bloc morphologique aux. + p.p. en français avant et après le lexème verbal est constante. Soit:

$$
\begin{array}{lll}
\text { Il est parti. } & \text { est }+ \text { part- } & + \text { i } \\
\text { Ils sont partis. } & \text { sont }+ \text { part- } & + \text { is } \\
\text { morphème } & +\downarrow_{\text {lexème }}+\text { morphème } \\
\text { aspectuel } & \text { verbal }
\end{array}
$$

En igbo, bien que la présence du marqueur aspectuel soit constante, celle de la voyelle d'harmonie ne l'est pas, comme l'atteste la traduction ci-dessous:

$$
\begin{aligned}
& \text { Opugo. } \\
& \text { Faapugo. } \\
& \begin{array}{c}
\text { voyelle + lexème } \\
\text { d'harmonie }
\end{array} \text { verbal }
\end{aligned}
$$

La configuration du marqueur aspectuel avec le préfixe d'harmonie n'est pas automatique; elle dépend plutôt du sujet, le verbe portant ainsi un préfixe d'harmonie seulement lorsque le sujet est nom, groupe nominal ou pronom pluriel, ou quand le verbe est participe. L'erreur de traduction serait d'hypergénéraliser la présence du préfixe harmonisant en suivant servilement la structure syntaxique française.

\subsection{L'obstacle lexico-syntaxique}

Comme pour la combinaison des morphèmes que l'on vient d'étudier, la syntaxe des éléments lexicaux d'aspect français ne peut pas être transportée directement à celle des marqueurs aspectuels igbo. Dans l'exemple:
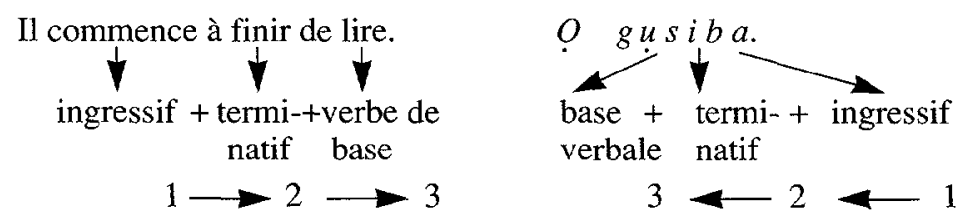
Il est clair que la séquence de marqueurs aspectuels n'est pas la même dans les deux langues. La combinaison des marqueurs français, qui sont d'ordre lexical, suit le mouvement prospectif; celle des marqueurs igbo, qui sont de nature morphémique, suit le mouvement rétrospectif, ce qui fait que l'ordre spatial d'aspectualisation est renversé. Cet ordre est légèrement modifié en igbo lorsque la combinaison comporte le marqueur d'imminence dans les deux langues:

Il va commencer à finir de lire.

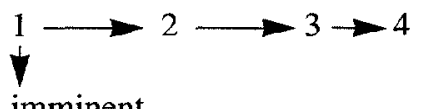

o gàfu à gu siba.

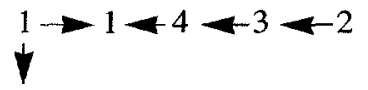

imminent

Pourtant, lorsque le premier élément lexical de la combinatoire aspectuelle française est grammaticalisé, l'ordre chiasmique qui a été constaté au niveau de la traduction de la première phrase se trouve renforcé :
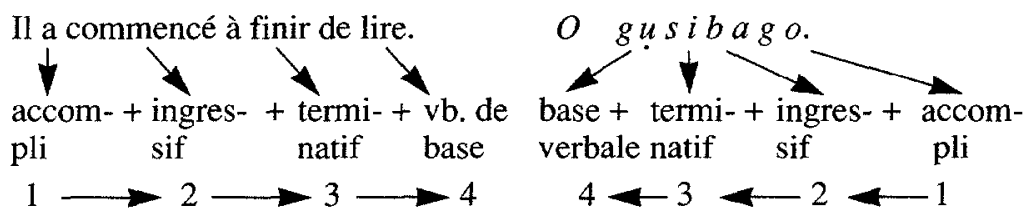

Pour éviter l'erreur de traduction, il faut connaître l'ordre syntaxique que suivent les marqueurs aspectuels aussi bien dans la langue-source que dans la langue-cible.

\subsection{L'obstacle sémantique}

Il arrive dans un cas extrême, voire imprévu, que les obstacles linguistiques dépassent le cadre structural et que le problème de la traduction persiste même après l'élimination de tout obstacle structuro-sémantique. C'est ce qui se produit lorsqu'un signifié de la langue-source n'existe pas ou existe partiellement dans la langue-cible ou bien inversement. Pour notre sujet, c'est le deuxième phénomène qui se produit: là où le français ne voit qu'une seule valeur aspectuelle l'igbo en voit deux à la fois, lesquelles coexistent simultanément et de façon indissociable, comme le révèle l'exemple ci-après:

Pierre continue à lire.

continuatif

\section{Pità nà àgugide akwukwo}

progressif + continuatif

Il en est ainsi, car je ne peux pas dire en igbo «Pità àgugide akwukwọ» en situant ainsi le morphème gide au présent dans la cooccurrence de nà. Inversement, «Pierre est en train de continuer à lire» est agrammatical. De là le décalage dans la quantité d'information véhiculée par les deux langues. La méconnaissance de l'importance sémantique de l'auxiliaire nà aboutirait ici à une phrase agrammaticale en igbo.

Les obstacles linguistiques ayant été localisés et reconnus en tant que tels, comment alors les éliminer?

\section{LE MÉCANISME DE TRADUCTION}

$\mathrm{Si}$, comme le disent justement Taber et Nida (1971: 11), «la traduction consiste à reproduire dans la langue réceptrice le message de la langue-source», il s'ensuit que la meilleure façon de franchir les obstacles linguistiques, et donc de rendre le message avec exactitude, est d'élaborer un mécanisme qui soit capable de dominer les servitudes 
linguistiques, mais aussi de rendre compte de ce qui peut être à la rigueur la différence d'apport d'information dans la même situation de communication. L'élaboration d'un tel mécanisme s'apparente à la construction d'un pont pour relier deux villes séparées d'un fleuve. Or, ce mécanisme est étroitement lié aux facteurs qui régissent la production des éléments linguistiques d'aspect dans chacune des deux langues, et qui en même temps facilitent leur traduction. Il s'agit précisément de phénomène sémantique. C'est ainsi que nous retiendrons la définition laroussienne (1964: 328) de l'aspect, à savoir, «l'angle sous lequel le parleur voit les différents moments du déroulement de l'action», à cette différence que cet angle de voir, c'est-à-dire la sémantique, dépend, lui aussi, d'un deuxième phénomène, de ce que Georges Mounin appelle «visions du monde ${ }^{5}$, et que nous préférerions appeler phénomène sémantico-vision. De même qu'au lieu de suivre l'orientation effet-cause comme l'a fait la Grammaire Larousse..., nous suivrons celle de cause à effet, celle du producteur au produit, celle de ce qui traduit à ce qui est traduit.

S'il est convenu que la traduction de l'aspect verbal français en igbo est d'abord une activité de production, puis celle de transfert, la traduction de l'aspect suivra alors le mécanisme suivant. Dans la langue source, le phénomène sémantique crée des phénomènes de nature sémantique : l'aspect inaccompli et l'aspect accompli, l'aspect étant inaccompli lorsque l'action n'est pas explicitement achevée, et accompli lorsque l'action est explicitement achevée. L'inaccompli et l'accompli créent à leur tour d'autres phénomènes à caractère sémantique: aspects divers, qui finalement créent les éléments linguistiques d'expression aspectuelle. Ces éléments linguistiques sont par la suite transférés à la langue-cible qui les reçoit par le même réseau de création sémantique, le point final de réception étant également les éléments linguistiques de LC. Ce réseau de transfert et de réception est véhiculé par le parallélisme sémantique entre LS et LC indépendamment de leur divergence structurale. Ainsi, si l'aspect est inaccompli (momentané, progressif, etc.) en LS, il est inaccompli en LC. Si, d'autre part, il est accompli en LS (accompli, ingressif accompli, etc.), il en est de même pour LC. Le mécanisme sémantico-vision suit le même processus de création sémantique, à cette différence près que sa création est limitée à l'inaccompli et que par conséquent l'opération de transfert se fait par le parallélisme sémantique partiel, ce qui se rapproche de ce que Vinay et Darbelnet (1958: 53) appellent adaptation ou équivalence de situation. Autrement dit, le mécanisme de traduction aspectuelle du français en igbo est le procédé selon lequel le traducteur, tout en envisageant le déroulement de l'action verbale, transforme, dans un premier temps, les éléments grammaticaux et lexicaux de LS en notions d'inaccompli et d'accompli, son choix étant déterminé par le sémantisme desdits items lexico-grammaticaux, puis l'action verbale tranfère le message à $L C$, ce message étant reçu dans $L C$ au moyen de l'auxiliaire à ton bas et surtout des morphèmes suffixaux à ton haut.

$\begin{array}{ll}\longrightarrow \quad \begin{array}{l}\text { Seront utilisés dans notre résumé les signes et sigles suivants : } \\ \text { crée }\end{array} \\ \text { se traduit } \\ + & \text { plus } \\ / & \text { ou } \\ \text { V } & \text { base verbale } \\ \text { VM } & \text { verbe momentané } \\ \text { Inf } & \text { infinitif } \\ \text { ØInf } & \text { infinitif zéro } \\ \text { AI } & \text { auxiliaire imminent } \\ \text { AIn } & \text { auxiliaire ingressif } \\ \text { AP } & \text { auxiliaire progressif } \\ \text { VP } & \text { verbe progressif }\end{array}$




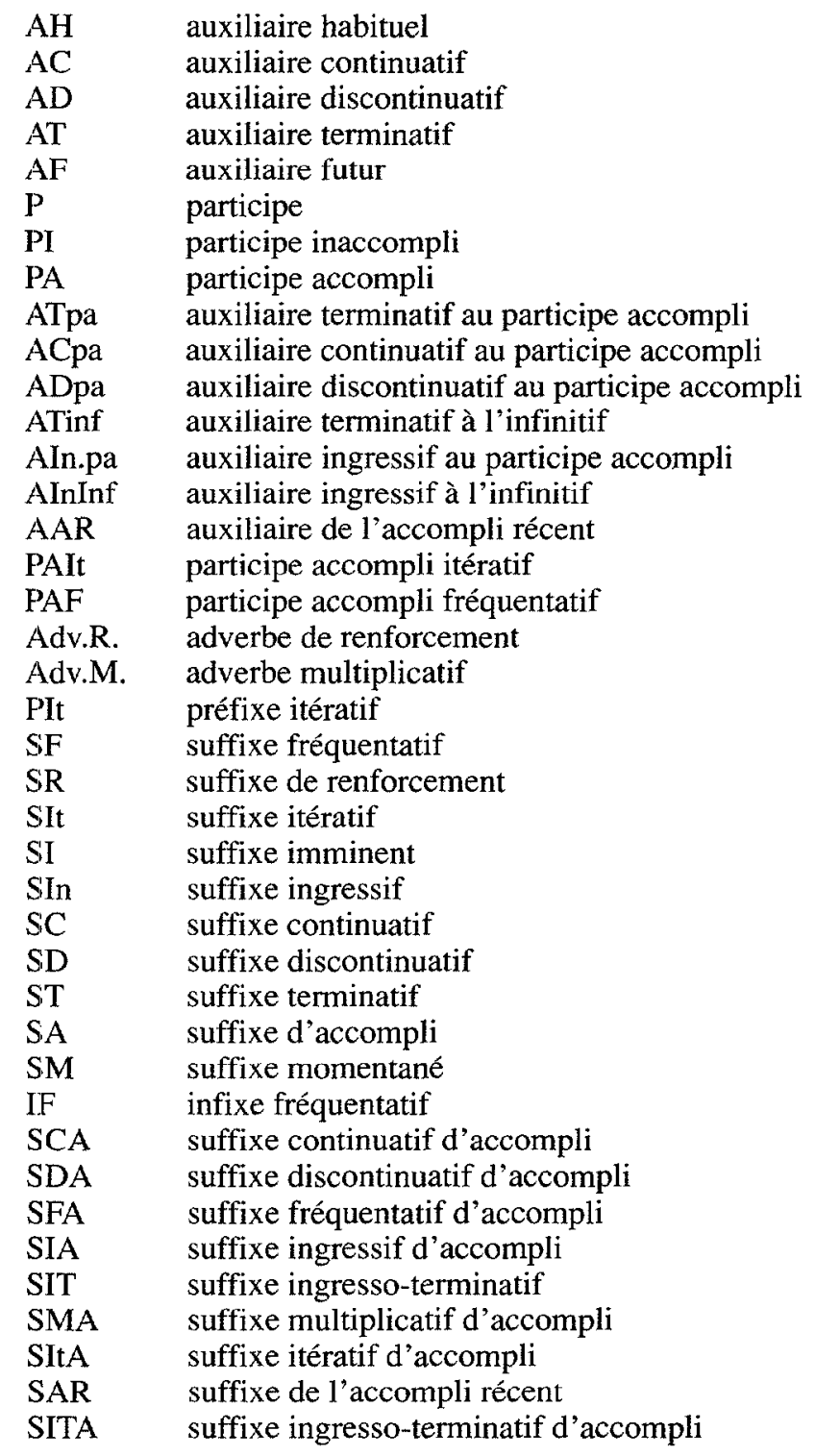

Auxiliaire veut dire auxiliaire, semi-auxiliaire, ou locution auxiliationnelle.

3.1 Mécanisme sémantique (visions du monde identiques, traduction par parallélisme sémantique)

\subsection{1 $\rightarrow$ Aspect inaccompli}

$\rightarrow$ momentané, imminent, ingressif, progressif, progressif renforcé, itéroprogressif, fréquento-progressif, habituel, discontinuatif, terminatif, terminatif imminent, ingresso-terminatif imminent. 
3.1.1.1 Momentané $\longrightarrow \mathrm{VM}: \mathrm{V}+\mathrm{SM}(-\mathrm{a})$

$\longrightarrow$ Le livre tombe. $\quad A k w u k w o$ àdàa.

3.1.1.2 Imminent $\rightarrow$ AI + Inf: $\mathrm{AF}(\mathrm{ga})+\mathrm{SI}(-\mathrm{fu})+\mathrm{P}$

Pierre va lire. Pità gàfu àgu akwukwo.

ou Pierre est sur le point de lire. Pità gàfu àgụ $a k w u k w o$.

3.1.1.3 Ingressif $\rightarrow$ AIn. + Inf: $\mathrm{V}+\mathrm{SIn}(-\mathrm{ba})$

$\longrightarrow$ Pierre commence à lire. Pità àguba akwıłkwo.

3.1.1.4 Progressif $\rightarrow$ AP+Inf/PI ou VP + ØInf: AP + P

$\longrightarrow$ Pierre est en train de lire. Pità nà àgu akwukwo.

ou Pierre lit. Pità nà àgu akwikwo.

ou Le petit chat va grelottant. Nwaobàrà nà àma jìjijịi.

3.1.1.5 Progressif renforcé $\longrightarrow$ AP+Inf+Adv.R : AP+P+SR(-lili)

$\longrightarrow$ Pierre est toujours en train de lire. Pità nà àgulili.

ou Pierre lit toujours. Pità nà àgulili.

3.1.1.6 Itéro-progressif $\longrightarrow \mathrm{PIt}+\mathrm{VP}: \mathrm{AP}+\mathrm{P}+\mathrm{SIt}(-\mathrm{zi})$

$\longrightarrow$ Pierre relit ce livre.

Pità nà àguzi akwrkwo afù.

3.1.1.7 Fréquento-progressif $\rightarrow \mathrm{VP}+\mathrm{IF}: \mathrm{AP}+\mathrm{P}+\mathrm{SF}$ (-cha)

$\rightarrow$ Les vautours tiraillent la charogne. Ùdènè nà àdocha ozu anù.

3.1.1.8 Habituel $\rightarrow \mathrm{AH}+\mathrm{Inf}: \mathrm{AH}+\mathrm{P}$

$\longrightarrow$ Pierre a l'habitude de lire. Pità nà àgụ akwukwo.

3.1.1.9 Discontinuatif $\longrightarrow$ AD + Inf: V + SD(-debe)

$\rightarrow$ Pierre cesse de lire. Pità àgudebe akwukwo.

3.1.1.10 Terminatif $\rightarrow$ AT + Inf: V + ST(-sia)

$\longrightarrow$ Pierre finit de lire. Pità àgusia akwrıkwo.

3.1.1.11 Terminatif Imminent $\rightarrow$ AI+ATinf+Inf: AF+SI(-fu)+P+ST(-si)

$\longrightarrow$ Pierre est sur le point de finir de lire. Pità gàfu àgusi.

3.1.1.12 Ingresso-terminatif imminent $\rightarrow$ AI+AIn.inf + ATinf + Inf $:$ AF+SI(fu)+P+SIT(-siba)

$\longrightarrow$ Pierre va commencer à finir de lire. Pità gàfu àgusiba.

\subsubsection{Aspect accompli}

$\longrightarrow$ accompli, ingressif accompli, continuatif accompli, discontinuatif accompli, itératif accompli, fréquentatif accompli, multiplicatif accompli, terminatif accompli, accompli récent, discontinuatif accompli futur, ingresso-terminatif accompli, ingressoterminatif accompli futur.

3.1.2.1 Accompli $\rightarrow$ avoir/être +PA: V+SA(-go)

$\longrightarrow$ Pierre a lu un livre. Pità àgugo akwukwo.

ou Marie est allée au marché. Marià èjego afia.

3.1.2.2 Ingressif accompli $\rightarrow$ avoir+AIn.pa + Inf: V+SIA(-bago)

$\longrightarrow$ Pierre a commencé à lire. Pità àgụbago akwukwo.

3.1.2.3 Continuatif accompli $\rightarrow$ avoir+ACpa+Inf: V+SCA(-gidego)

$\longrightarrow$ Pierre a contiué à lire. Pità àgugidego. 


\subsubsection{Discontinuatif accompli $\longrightarrow$ avoir+ADpa+Inf: V+SDA(-debago)}

$\rightarrow$ Pierre a cessé de lire. Pità àgụdebago.

3.1.2.5 Itératif accompli $\longrightarrow$ avoir+PAIt: V + SItA(-zigo)

$\rightarrow$ Pierre a relu un livre. Pità àguzigo akwukwo.

3.1.2.6 Fréquentatif accompli $\rightarrow$ avoir + PAF: V + SFA(-chago)

$\longrightarrow$ Les vautours ont tiraillé la charogne. Ùdènè àdochago ozu anù.

3.1.2.7 Multiplicatif accompli $\rightarrow$ avoir+PA+Adv.M: V+SMA(katago)

$\rightarrow$ Pierre a lu plusieurs fois. Pità àgukatago akwukwo.

3.1.2.8 Terminatif accompli $\rightarrow$ avoir + ATpa + Inf : V + STA(-sigo)

$\longrightarrow$ Pierre a fini de lire. Pità àgusigo akwukwo.

3.1.2.9 Accompli récent $\rightarrow$ AAR + Inf: $\mathrm{V}+\mathrm{SAR}(-\mathrm{fuu})$

$\longrightarrow$ Pierre vient de lire. Pità gựụu akwukwo.

3.1.2.10 Discontinuatif accompli futur $\rightarrow \mathrm{AF}+\mathrm{AD}$.pa+Inf: $\mathrm{AF}+\mathrm{P}+\mathrm{SDA}$ (debago)

$\rightarrow \quad$ Pierre aura cessé de lire. Pità gà àgudebago akwukwo.

3.1.2.11 Ingresso-terminatif accompli $\rightarrow$ avoir + AInpa+ATinf+Inf: V + SITA(-sibago)

$\rightarrow$ Pierre a commencé à finir de lire. Pità àgusibago.

3.1.2.12 Ingresso-terminatif accompli futur $\rightarrow$ AF+AIn.pa+ATinf + Inf: $\mathrm{AF}+\mathrm{V}+\mathrm{SITA}$ (-sibago)

$\longrightarrow$ Pierre aura commencé à finir de lire. Pità gà àgusibago.

3.2 Mécanisme sémantico-vision (Visions du monde partiellement différentes, traduction par parallélisme quasi sémantique, quasi situationnel)

3.2.1 $\rightarrow$ Aspect inaccompli

$\rightarrow$ continuatif: continuatif progressif,

$\rightarrow$ multiplicatif : multiplicatif progressif.

3.2.1.1 Continuatif: Continuatif progressif $\rightarrow \mathrm{AP}+\mathrm{inf}: \mathrm{AP}+\mathrm{V}+\mathrm{SC}$ (-gide)

$\longrightarrow$ Pierre continue à lire. $\quad$ Pità nà àgugide $a k w u k w o$.

3.2.1.2 Multiplicatif: Multiplicatif progressif $\rightarrow \mathrm{V}+\mathrm{Adv} \cdot \mathrm{M}: \mathrm{AP}+\mathrm{V}+\mathrm{SM}(-\mathrm{kata})$

$\rightarrow$ Pierre lit plusieurs fois. Pità nà àgukata akwukwo.

CONCLUSION

Cette étude a montré que toutes les expressions aspectuelles françaises peuvent se traduire facilement en igbo, mais que l'igbo n'utilise pas de système linguistique identique dans cette opération. Ce que le français exprime essentiellement par les éléments désinentiels et lexicaux, «tif», l'igbo traduit principalement par des morphèmes suffixaux et par les tons. Grâce au parallélisme sémantique qui existe entre les deux langues dans leur processus de création aspectuelle, il a été possible de lever les écueils structuraux et donc de traduire. C'est la raison pour laquelle l'aspect qui a été inaccompli ou accompli en français l'a été en même temps en igbo. C'est pourquoi, à un niveau plus haut de la correspondance sémantique, les aspects ingressif, discontinuatif et terminatif qui ont été possibles en français aussi bien à l'inaccompli qu'à l'accompli l'ont été également en igbo (vide 3.1.1.3 et 3.1.1.2, 3.1.1.9 et 3.1.2.4, 3.1.1.10 et 3.1.2.8 respectivement). Et inversement, c'est pourquoi les connotations aspectuelles d'imminence et de progressif qui se 
sont avérées inopératoires à l'accompli en LS se sont révélées du même coup impossibles à l'accompli en LC. Grâce au phénomène sémantico-vision (cf. 3.2), on a pu résoudre le problème de traduction des deux cas d'inaccompli récalcitrants qui ont échappé aux universaux sémantiques.

En outre, l'intérêt de cette traduction réside dans le fait qu'elle nous a permis d'apprécier le fonctionnement de LS et de LC mieux que nous n'aurions pu le faire dans un traitement unilingue. Pour n'en citer que quelques exemples, ce n'est qu'en traduisant en igbo «Pierre a lu un livre» qu'on est arrivé à être sensibilisé sur la véritable valeur sémantique de cette combinatoire complexe (aux. + pp), celle d'aspect plutôt que de temps, dans la mesure où en igbo la marque de l'aspect accompli est différente de celle $\mathrm{du}$ temps parfait aussi bien morphémiquement que tonalement. Ce n'est qu'en rendant «Pierre va lire un livre» en «Pità gàfu àgu akwrkwo» que la valeur aspectuelle de la phrase française se dégage mieux, celle d'imminence, en ce sens que l'igbo dispose d'une forme spécifique pour le futur proche aussi bien que pour le mouvement d'éloignement. L'étude nous a amené aussi à faire la distinction nette entre certains auxiliaires français qui sont de faux amis, ex. entre finir de et cesser de (terminatif et discontinuatif), alors que traditionnellement on les classe ensemble sous le tiroir du terminatif, et à les distinguer encore au niveau de la dénotation aspectuelle; or, quoique perfectifs implicitement, ils sont explicitement et réellement inaccomplis en 3.1.1.10 et 3.1.1.9 respectivement. De même qu'une opposition analogue a facilité la tâche de situer aspectuellement des combinatoires longues, ex. «Pierre a commencé à finir de lire», comme accomplies, la grammaticalisation du premier élément auxiliaire ou modal ayant prolongé l'ensemble de la combinatoire dans la sphère d'accompli ou, si on préfère, dans un accompli subjectif ou précisément dans la modalité d'accompli, cet accomplissement étant confirmé en igbo par le marqueur -go. Inversement, ce n'est qu'au milieu de ce travail qu'on est tombé par hasard sur l'ambiguïté aspectuelle de l'auxiliaire nà (cf. 2.4). Par ailleurs, il est passionnant d'avoir découvert les morphèmes - a (momentané), -zi (itératif), -cha (fréquentatif), -debe (discontinuatif), -sia (terminatif), et les morphèmes complexes suivants: -bago (3.1.2.2), -gidego (3.1.2.3), -debago (3.1.2.4), -zigo (3.1.2.5), -chago (3.1.2.6), -katago (3.1.2.7), -sigo (3.1.2.8) et -sibago (3.1.2.11), lesquels viennent compléter la liste de marqueurs aspectuels déjà signalés par Emenanjo (1978) et par SPILC (1985: 17, 238). Il faudrait une étude approfondie pour une meilleure appréciation du système aspectuel igbo.

Finalement, à noter que la correspondance sémantique quasi totale obtenue dans cette activité traduisante est due à la richesse de la langue igbo en aspect verbal, ce qui ne signifie nullement qu'on aurait eu le même résultat en traduisant d'autres catégories grammaticales françaises.

\section{BIBLIOGRAPHIE}

BRISLIN, Richard W. (1945): Translation, Application and Research, New York, Gardner Press Inc. CHEVALIER, J.-C., BLANCHE-BENVENISTE, C., ARRIVE, M., et J. PEYRARD (1964): Grammaire Larousse du français contemporain, Paris, Larousse.

EMENANJO, N. E. (1978): Elements of Modern Igbo Grammar, Ibadan, O.U.P.

LADMIRAL, J.-R. (1979) : Traduire : théorème pour la traduction, Paris, Petite bibliothèque Payot. MOUNIN, Georges (1963) : Les Problèmes théoriques de la traduction, Paris, Gallimard.

OGBALU, F. C. (1981): Onuogugu Igbo, Onitsha, University Publishing Co.

OKIWEMU, B. O. (1985) : «La traduction de l'impersonnel français en igbo», Autour de l'impersonnel, Paris, Ellug.

OKIWEMU, B. O. (1982) : «L'auxiliarisation en français et les problèmes de la traduction», AIT l'Interprète, $\mathrm{n}^{\circ} 4$. 
OKIWEMU, B. O. (1986): «Les marques prépositionnelles de l'auxiliarisation française et le mécanisme de leur traduction en anglais», AIT l'lnterprète, $\mathrm{n}^{\circ} 1$.

SPILC (Society for the Promotion of Igbo Language and Culture) (1985): Okaasusu Igbo, SPILC Nigeria.

TABER, C.-R., NIDA, E.-A. (1971): La Traduction: théorie et méthode, London, New York, Stuttgart, Alliance biblique.

VINAY, J.P. et J. DARBELNET (1958) : Stylistique comparée du français et de l'anglais, Paris, Didier. WIESEMMANN, Ursula (1986): Manuel de sémantique et de traduction, Yaoundé, collection Propelon ${ }^{\circ} 33$. 